\title{
Diaper dermatitis: a survey of risk factors in Thai children aged under 24 months
}

\author{
Chonnakarn Sukhneewat ${ }^{1}$, Jitjira Chaiyarit ${ }^{2}$ and Leelawadee Techasatian $^{3^{*}}$ (D)
}

\begin{abstract}
Background: To identify the prevalence and risk factors associated with diaper dermatitis in Thai children aged 1-24 months.

Methods: This was a cross-sectional study of 1153 participants using structural questionnaires, which was conducted at Khon Kaen University Faculty of Medicine Pediatric department in Thailand. Univariate and multivariate logistic regression analyses were used to test the association between diaper dermatitis and its possible risk factors.
\end{abstract}

Results: The prevalence of diaper dermatitis among the study population was $36.1 \%$, a rate which significantly decreased with age. The highest prevalence was found in subjects who were one to six months old. Risk factors that had a statistically significant association with diaper dermatitis in both univariate and multivariate analysis were i) diaper changing fewer than three times/night, ii) previous episodes of diaper rash, iii) using cloth diapers, and iv) topical application of baby talcum powder to the diaper area.

Conclusions: Frequent diaper changings during the daytime do not compensate for fewer changings during the night. Moreover, the use of baby talcum powder on the diaper area significantly increased the risk of diaper dermatitis among the study population. These findings should be applied in future preventive strategies for diaper dermatitis in this age group.

Keywords: Baby talcum powder, Dermatitis, Diaper dermatitis, Risk factor

\section{Background}

Diaper dermatitis refers to any clinical sign of skin inflammation that occurs on the area covered by a diaper. Most skin inflammation is irritation caused by moisture, heat, or various enzymes from urine and feces, which are major irritants to the skin [1-4]. There is a high prevalence of diaper dermatitis among children who require diapering. The estimated overall prevalence has been found to range from 7 to $43.8 \%$ and varies according to setting, hygiene practices, and age group [5]. Children under 24 months old have the highest prevalence, with the peak being between nine and 12 months of age. This is likely due to the fact that the children in this age group require diapering [3] at a greater rate than those in other age groups. There are many risk factors that have been found to increase the occurrence of diaper

\footnotetext{
* Correspondence: leelawadee@kku.ac.th

${ }^{3}$ Dermatology Division, Pediatric Department, Faculty of Medicine, Khon Kaen University, Khon Kaen 40002, Thailand

Full list of author information is available at the end of the article
}

dermatitis. These factors included gastrointestinal tract infection, the type of diaper used, and the frequency of diaper changes [6]. There is little information available on diaper dermatitis in Thailand. This study was, thus, developed to explore the prevalence and risk factors of diaper dermatitis among Thai children in this age group (1-24 months). The results from this present study will have application in the future development of preventive strategies for diaper dermatitis in Thai children.

\section{Methods}

This cross-sectional study was conducted at the Khon Kaen University, Faculty of Medicine, Pediatric Department, in the Well Baby Clinics between November 2015 and January 2017. All children aged 1-24 months were eligible.

Consecutive cases entered into the Well Baby Clinics were asked to participate in the study. A total number of 1153 children were enrolled based on our sample size 
calculation. The diagnosis of diaper dermatitis was made based on parental reporting of any skin rashes on the diaper area during the past 6 weeks. The demographic background information included in the structural questionnaires was age, sex, general condition, and underlying atopic diseases. The possible risk factors of diaper dermatitis, including gastrointestinal tract infection, type of diaper used, and frequency of diaper changes, were also addressed in a structural questionnaire. A detailed description of the questionnaire is included in an Additional file 1.

At the end of the study, the collected data were analyzed using STATA software version 10 (StataCorp LP). Descriptive statistical methods - means, standard deviations (SDs), medians and frequencies - were used to analyze the demographic data. Univariate and multivariate logistic regression analyses were performed to test the associations between the proposed factors and diaper dermatitis. The authors first estimated the association between each risk factor and diaper dermatitis (bivariate analysis). The initial multivariate analysis model included all risk factors. Independent risk factors for diaper dermatitis were identified by forward stepwise logistic regression where all univariate predictors with a $p$-value $<0.05$ were included. Multivariate regression was corrected for multiple testing. Values of $P<0.05$ were considered to indicate statistical significance. Incomplete questionnaires and missing data were addressed as imputed data, and final calculations included all recorded data.

The study was approved by the institutional review board of the Khon Kaen University, Human Ethical Committee (\#HE581286). Before participants were enrolled in the study, written informed consent was obtained from their parents or guardians. The study was funded by a grant from the Khon Kaen University, Faculty of Medicine in Thailand: (Grant Number IN58332).

\section{Results}

A total of 1153 children were recruited for the study: 585 (50.7\%) boys and 568 (49.3\%) girls. Their ages ranged from 1 to 24 months (median = six months) with a mean age of 8.7 months (SD 4.3). The prevalence of diaper dermatitis was $36.1 \%(416 / 1153)$. The highest prevalence was among the children who were 1-6 months old (47.9\%). This age group also had a significantly higher prevalence of diaper dermatitis than the other three age groups (7-12, 13-18 and 19-24 months; $P<0.05)$. Figure 1 represents the variation in the prevalence of diaper dermatitis among the different age groups. There were no significant differences in term of prevalence between sexes (boys 37.4\% [219/585] versus girls $34.5 \%$ [249/721]; $P$-value $=0.31$.

Univariate analysis showed that diaper dermatitis was significantly associated with the following five factors: i) underlying allergic diseases, ii) fewer than three diaper changes per night, iii) previous episodes of diaper rash, iv) use of cloth diapers, and v) use of baby talcum powder on the diaper area when diaper dermatitis is present (Table 1). Four of the five factors that were significantly associated with diaper dermatitis according to univariate logistic regression analysis were also significantly related according to multivariate logistic regression analysis. (factors ii trough v; $P<0.001$ for all comparisons; Table 2).

Underlying allergic diseases (atopic dermatitis, allergic rhinitis, asthma and cow milk protein allergies) were documented as having significant association with diaper dermatitis according to univariate logistic regression analysis. However, further multivariate logistic regression calculation could not be performed for each individual allergic condition because the sample size in each of these groups was too small. Other factors that were listed in the structural questionnaires but had no

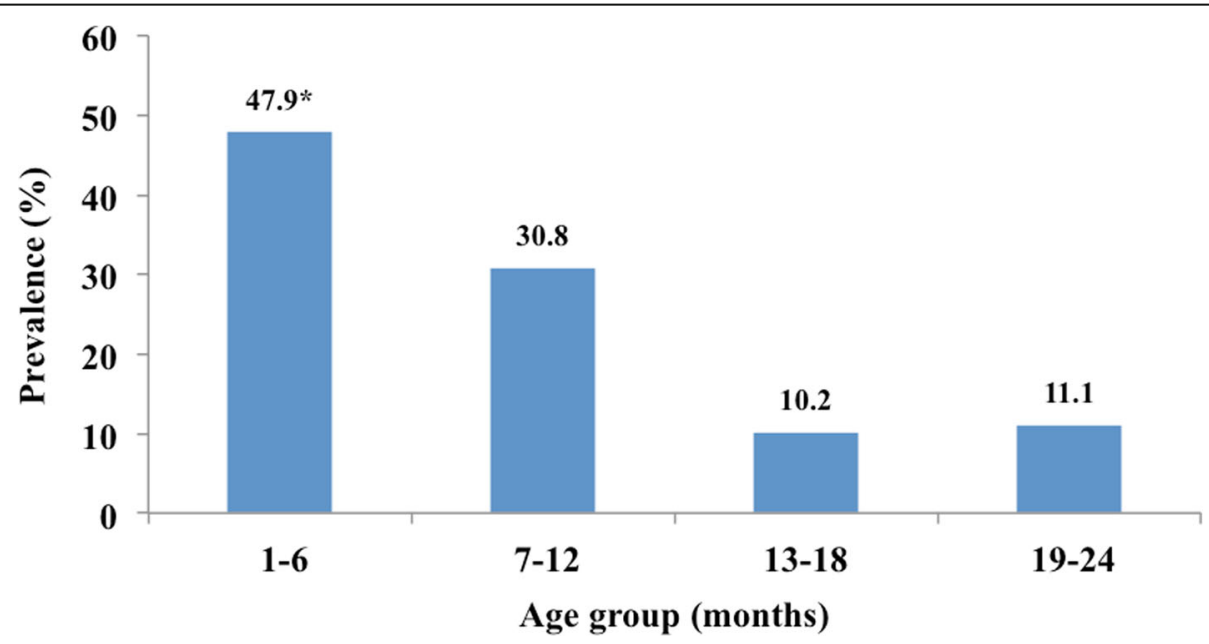

Fig. 1 Variation in the prevalence of diaper dermatitis among different age groups 
Table 1 Summary of the factors associated with diaper dermatitis in the study population

\begin{tabular}{|c|c|c|c|c|c|}
\hline \multirow[t]{2}{*}{ Factors } & \multicolumn{2}{|c|}{ Diaper dermatitis } & \multicolumn{3}{|c|}{ Univariate logistic regression } \\
\hline & Yes $n=416$ & No $n=737$ & $\overline{O R}$ & $95 \% \mathrm{Cl}$ & $P$-value \\
\hline Age & & & & & $<0.001^{*}$ \\
\hline $1-6$ months & $239(47.99)$ & $259(52.01)$ & 1 & $0.37,0.64$ & \\
\hline 7-12 months & $122(30.81)$ & $274(69.19)$ & 0.48 & $0.18,0.41$ & \\
\hline 13-18 months & $35(20.23)$ & $138(79.77)$ & 0.28 & $0.19,0.56$ & \\
\hline 19-24 months & $20(23.26)$ & $66(76.74)$ & 0.33 & $0.37,0.64$ & \\
\hline Children with underlying allergic diseases & & & & & $<0.001^{*}$ \\
\hline No & $317(33.54)$ & $628(66.46)$ & 1 & & \\
\hline Yes & $99(47.60)$ & $109(52.40)$ & 1.8 & $1.33,2.44$ & \\
\hline Type of diaper using & & & & & $<0.001^{*}$ \\
\hline Disposable diaper [40.4\% (466/1153)] & $79(16.95)$ & $387(83.05)$ & 1 & & \\
\hline Cloth diaper [59.6\% (687/1153)] & $337(49.05)$ & $350(50.95)$ & 4.72 & $3.55,6.27$ & \\
\hline Previous episodes of diaper dermatitis & & & & & $<0.001^{*}$ \\
\hline None & $19(6.96)$ & $254(93.04)$ & 1 & & \\
\hline$\leq 2$ times & $258(51.09)$ & $247(48.91)$ & 13.96 & $8.49,22.97$ & \\
\hline$>2$ times & $139(37.07)$ & $236(62.930$ & 7.87 & $4.72,13.13$ & \\
\hline \multicolumn{6}{|l|}{ Frequency of diaper changes } \\
\hline Total number/day & & & & & 0.717 \\
\hline$\geq 6$ times & $159(35.49)$ & $289(64.51)$ & 1 & & \\
\hline$<6$ times & $254(36.55)$ & $441(63.45)$ & 1.05 & $(0.82,1.34)$ & \\
\hline Total number/daytime & & & & & 0.765 \\
\hline$\geq 3$ times & $155(35.63)$ & $280(64.37)$ & 1 & & \\
\hline$<3$ times & $261(36.50)$ & $454(63.50)$ & 1.04 & $0.81,1.33$ & \\
\hline Total number/nighttime & & & & & $<0.001^{*}$ \\
\hline$\geq 3$ times & $16(12.21)$ & $115(87.79)$ & 1 & & \\
\hline$<3$ times & $387(38.82)$ & $610(61.18)$ & 4.56 & $(2.66,7.81)$ & \\
\hline Topical application on diaper area & & & & & $<0.001^{*}$ \\
\hline Diaper cream & $46(29.30)$ & $111(70.70)$ & 1 & & \\
\hline Baby powder & $173(40.42)$ & $255(59.58)$ & 1.64 & $1.10,2.43$ & \\
\hline Baby lotion & $9(17.31)$ & 43 (82.69) & 0.51 & $0.23,1.12$ & \\
\hline
\end{tabular}

association with increased prevalence of diaper dermatitis were parental age, parental education, child's weight, and type of food consumed during the past 6 weeks.

\section{Discussion}

To our knowledge, this study is the first report on the prevalence of diaper dermatitis in Thai children. The age of the study population ranged from 1 to 24 months, as previous studies have found children in this age range to have a high prevalence of diaper dermatitis [5, 7-9]. The high prevalence is due to the fact that children in this age range still need diapering. Most children over two years of age are able to use the toilet by themselves, decreasing the need for diapering substantially.

The prevalence of diaper dermatitis in the present study was $36.1 \%$. This number was similar to those found in previous studies, which have ranged from 7 to $43.8 \%$. There was no difference in the prevalence of diaper dermatitis between boys and girls, a finding that is similar to those of other studies in Asian [10], North American, and European countries [5, 9, 11].

The present study classified the children into four age groups: $1-6,7-12,13-18$ and 19-24 months. The highest prevalence was found among the children aged 1-6 months with a significantly higher prevalence than the other three age groups $(P<0.001)$.

The standard recommendation is that diapers should be changed every three to four hours, a duration that is based on the frequency of urination in infants $[1-3,12]$. This means that diapers should be changed from six to eight times/day. This is one of the major factors that influence the prevalence of diaper dermatitis, as the 
Table 2 Summary of the five factors that were found to be significantly associated with diaper dermatitis in Thai children aged 124 months according to multivariate logistic regression analysis

\begin{tabular}{|c|c|c|c|c|c|}
\hline \multirow[t]{2}{*}{ Factors } & \multicolumn{2}{|c|}{ Univariate logistic regression } & \multicolumn{3}{|c|}{ Multivariate logistic regression } \\
\hline & $\mathrm{OR}$ & $95 \% \mathrm{Cl}$ & $\overline{\mathrm{OR}}$ & $95 \% \mathrm{Cl}$ & $P$-value \\
\hline \multicolumn{6}{|l|}{ Age } \\
\hline $1-6$ months & 1 & & 1 & & \\
\hline 7-12 months & 0.48 & $0.37,0.64$ & 0.35 & $0.25,0.49$ & $<0.001^{*}$ \\
\hline 13-18 months & 0.28 & $0.18,0.41$ & 0.15 & $0.09,0.24$ & $<0.001^{*}$ \\
\hline 19-24 months & 0.33 & $0.19,0.56$ & 0.31 & $0.17,0.58$ & $0.001^{*}$ \\
\hline \multicolumn{6}{|l|}{ Type of diaper using } \\
\hline Disposable diaper & 1 & & 1 & & \\
\hline Cloth diaper & 4.72 & $3.55,6.27$ & 5.47 & $3.78,7.92$ & $<0.001^{*}$ \\
\hline \multicolumn{6}{|c|}{ Previous episodes of diaper dermatitis } \\
\hline None & 1 & & 1 & & \\
\hline$\leq 2$ times & 13.96 & $8.49,22.97$ & 12.16 & $6.72,22.01$ & $<0.001^{*}$ \\
\hline$>2$ times & 7.87 & $4.72,13.13$ & 9.71 & $5.28,17.86$ & $<0.001^{*}$ \\
\hline \multicolumn{6}{|c|}{ Topical application on diaper area } \\
\hline Diaper cream & 1 & & 1 & & \\
\hline Baby powder & 1.64 & $1.10,2.43$ & 1.61 & $1.04,2.57$ & $0.005^{*}$ \\
\hline Baby lotion & 0.51 & $0.23,1.12$ & 2.59 & $1.37,9.39$ & $0.009^{*}$ \\
\hline \multicolumn{6}{|c|}{ Total number of diaper changing/nighttime } \\
\hline$\geq 3$ times & 1 & & 1 & & \\
\hline$<3$ times & 4.56 & $(2.66,7.81)$ & 3.72 & $2.04,6.76$ & $<0.001^{*}$ \\
\hline
\end{tabular}

condition becomes more likely when there is prolonged contact of urine and feces with the skin. Previous studies have shown that the prevalence of diaper dermatitis is significantly higher when diapers are changed fewer than six times/day compared with more frequent changing [5]. The present study went further and compared the effects of daytime changings versus nighttime changings on the prevalence of diaper dermatitis. We found that fewer than three changes/night led to a significant increase in the risk of diaper dermatitis compared to more frequent changings (multivariate logistic regression analysis, $\mathrm{OR}=3.72, \quad P<0.001)$. However, fewer than three changings during the daytime did not lead to significant increases in diaper dermatitis, meaning that more frequent daytime changings cannot compensate for fewer changings during the night. These findings support the idea that it is important to change an infant's diaper after each time he/she urinates.

In the present study, 40.4\% (466/1153) of participants used only disposable diapers, and $59.6 \%$ $(687 / 1153)$ used only cloth diapers. The authors found that the rate of diaper dermatitis was significantly higher in the participants who used cloth diapers compared to those who used disposable diapers, (multivariate analysis $\mathrm{OR}=5.47, \quad P<0.001$ ). This is consistent with the results of previous studies conducted after absorbent gel technology had become widely used in disposable diapers [9, 13-15].

Although a Cochrane review [16] could not find enough evidence from good-quality randomized controlled trials to support or refute the benefit of disposable diapers in the prevention of diaper dermatitis in infants, their use is recommended for all infants. This is supported by evidence that a new absorbent gel technology in disposable diapers is effective in drawing urine away from the diaper area and keeping the skin dry, reducing dermatological problems in the diaper area [17-19].

All of the disposable diaper brands used by participants in this study (MamyPoko, BabyLove, Goon, and Huggies), used the newly developed absorbent gel technology mentioned above. This may explain the significantly lower rate of diaper dermatitis in participants who used disposable diapers compared to those who used cloth diapers.

It is well known that using diaper creams that enhance the skin barrier is an effective strategy for preventing diaper dermatitis [20-22]. The major properties of overthe-counter diaper creams that help prevent diaper rash are as follows: i) containing fragrance-free moisturizer to restore skin barrier function, ii) exhibiting a barrier effect to protect the skin from major irritants (urine, 
feces), and iii) containing dexpanthenol (B5), which has been proven to be effective in treating skin inflammation on the diaper area $[21,22]$. In Thailand, there are many effective diaper creams that are readily available over the counter. However, baby talcum powder is more frequently used for this purpose. The present study showed that, 37.1\% (428/1153) of the study population used baby powder on the diaper area, with only $13.6 \%(157 / 1153)$ using an effective diaper cream.

Topical application of baby talcum powder is thought to be useful in keeping the skin dry [11, 23, 24]. However, it has only been found to be beneficial in cases of uncomplicated diaper dermatitis and not in more severe cases of cutaneous inflammation [25]. In addition, the application of talcum powder to areas of the skin where the epidermal barrier is absent or significantly disrupted or to moist areas can cause skin irritation [13, 15, 26]. The most serious possible side effect of baby talcum powder that has been reported is respiratory distress from accidental massive aspiration [27]. Moreover, another report found that prolonged inhalation of talcum powder can induce surfactant depletion, bronchiolar hyper-responsiveness, and alveolar damage [28]. According to these reports, baby talcum powder should be used only to preserve skin dryness to a limited extent on certain areas. The present study also found that the use of baby talcum powder significantly increased diaper dermatitis when compared to diaper creams in the study population (multivariate logistic regression analysis, $\mathrm{OR}=1.61, P=0.005)$. Thus, the use of diaper creams rather than baby talcum powders should be encouraged in the Thai population.

There were several potential limitations to the present study. The questionnaire was only structured to examine possible risk factors for diaper dermatitis. However, there may be other unknown factors that have an impact on diaper dermatitis that were not included in the analyses. In addition, some of the respondents did not complete the sections in the questionnaires regarding underlying atopic diseases and economic status. The authors addressed the missing information as imputed data and calculated the recorded data all together. This may have affected the power of the test, but the main possible risk factors were record completely.

\section{Conclusions}

Changing diapers fewer than three times/night and using baby talcum powder increased the risk of diaper dermatitis among the study population. Therefore, future strategies to prevent diaper dermatitis in this age group should emphasize frequent diaper changing (especially at night) and the application of diaper cream containing dexpanthenol (B5) instead of baby talcum powder on the diaper area.

\section{Additional file}

Additional file 1: A structural questionnaire. A detailed description of the questionnaire. (DOCX $66 \mathrm{~kb}$ )

\section{Acknowledgements}

Not applicable.

\section{Authors' contributions}

CS - performed studies and data collection. JC - analyzed data and prepared Figs. LT - conceptualized and designed the study and wrote the manuscript. All authors read and approved the final version of manuscript.

\section{Funding}

The study was funded by a grant from the Khon Kaen University, Faculty of Medicine, Thailand in the design of the study; collection, analysis, and interpretation of data; and in writing the manuscript (Grant Number IN58332).

\section{Availability of data and materials}

The datasets used and/or analysed during the current study are available from the corresponding author on reasonable request.

\section{Ethics approval and consent to participate}

The study was approved by the institutional review board of the Khon Kaen University, Human Ethical Committee (\#HE581286). Before participants were enrolled in the study, written informed consent was obtained from their parents or guardians.

Consent for publication

Not applicable.

\section{Competing interests}

The authors declare that they have no competing interests.

\section{Author details}

${ }^{1}$ Pediatric Department, Faculty of Medicine, Khon Kaen University, Khon Kaen, Thailand. ${ }^{2}$ Clinical epidemiology unit, Faculty of Medicine, Khon Kaen University, Khon Kaen, Thailand. ${ }^{3}$ Dermatology Division, Pediatric

Department, Faculty of Medicine, Khon Kaen University, Khon Kaen 40002, Thailand.

Received: 14 January 2019 Accepted: 22 May 2019

Published online: 02 July 2019

\section{References}

1. Atherton DJ. A review of the pathophysiology, prevention and treatment of irritant diaper dermatitis. Curr Med Res Opin. 2004;20(5):645-9.

2. Shin HT. Diagnosis and management of diaper dermatitis. Pediatr Clin N Am. 2014:61(2):367-82.

3. Berg RW. Etiology and pathophysiology of diaper dermatitis. Adv Dermatol. 1988;3:75-98.

4. Ravanfar P, Wallace JS, Pace NC. Diaper dermatitis: a review and update. Curr Opin Pediatr. 2012;24(4):472-9.

5. Li CH, Zhu ZH, Dai YH. Diaper Dermatitis: a survey of risk factors for children aged 1 - 24 months in China. J Int Med Res. 2012;40(5):1752-60.

6. Ward DB, Fleischer AB, Feldman SR, Krowchuk DP. Characterization of diaper dermatitis in the United States. Arch Pediatr Adolesc Med. 2000;154(9):943-6.

7. Singalavanija S, Frieden IJ. Diaper Dermatitis. Pediatr Rev. 1995;16(4):142-7.

8. Adalat S, Wall D, Goodyear H. Diaper dermatitis-frequency and contributory factors in hospital attending children. Pediatr Dermatol. 2007;24(5):483-8.

9. Scheinfeld N. Diaper dermatitis: a review and brief survey of eruptions of the diaper area. Am J Clin Dermatol. 2005;6(5):273-81.

10. Prasad HRY, Srivastava P, Verma KK. Diaper dermatitis--an overview. Indian J Pediatr. 2003;70(8):635-7.

11. Thaman LA, Eichenfield LF. Diapering habits: a global perspective. Pediatr Dermatol. 2014;31:15-8.

12. Klunk C, Domingues E, Wiss K. An update on diaper dermatitis. Clin Dermatol. 2014;32(4):477-87. 
13. Coughlin CC, Frieden IJ, Eichenfield LF. Clinical approaches to skin cleansing of the diaper area: practice and challenges. Pediatr Dermatol. 2014;31 Suppl:1:1-4.

14. Odio M, Friedlander SF. Diaper dermatitis and advances in diaper technology. Curr Opin Pediatr. 2000;12(4):342-6.

15. Humphrey S, Bergman JN, Au S. Practical management strategies for diaper dermatitis. Skin Ther Lett. 2006;11(7):1-6.

16. Baer EL, Davies MW, Easterbrook K. Disposable nappies for preventing napkin dermatitis in infants [Internet]. In: Cochrane Database of Systematic Reviews. John Wiley \& Sons, Ltd; 2006 [cited 2017 Jul 14]. Available from: http://onlinelibrary.wiley.com/doi/10.1002/14651858.CD004262.pub2/ abstract

17. Odio M, Thaman L. Diapering, diaper technology, and diaper area skin health. Pediatr Dermatol. 2014;31(Suppl 1):9-14.

18. Burdall O, Willgress L, Goad N. Neonatal skin care: developments in care to maintain neonatal barrier function and prevention of diaper dermatitis. Pediatr Dermatol. 2019;36(1):31-5.

19. Blume-Peytavi U, Hauser M, Lünnemann L, Stamatas GN, Kottner J, Garcia Bartels N. Prevention of diaper dermatitis in infants--a literature review. Pediatr Dermatol. 2014;31(4):413-29.

20. Wananukul S, Limpongsanuruk W, Singalavanija S, Wisuthsarewong W. Comparison of dexpanthenol and zinc oxide ointment with ointment base in the treatment of irritant diaper dermatitis from diarrhea: a multicenter study. J Med Assoc Thail Chotmaihet Thangphaet. 2006;89(10):1654-8.

21. Proksch E, Nissen HP. Dexpanthenol enhances skin barrier repair and reduces inflammation after sodium lauryl sulphate-induced irritation. J Dermatol Treat. 2002;13(4):173-8.

22. Ebner F, Heller A, Rippke F, Tausch I. Topical use of dexpanthenol in skin disorders. Am J Clin Dermatol. 2002;3(6):427-33.

23. Goldman M, Lodhi I. A real-world evidence study evaluating a treatment for nappy rash. Br J Nurs Mark Allen Publ. 2016;25(8):432-9.

24. Scheinfeld DN. Diaper Dermatitis. Am J Clin Dermatol. 2012;6(5):273-81.

25. Fiume MM, Boyer I, Bergfeld WF, Belsito DV, Hill RA, Klaassen CD, et al. Safety assessment of talc as used in cosmetics. Int J Toxicol. 2015:34(1_suppl:66S-129S.

26. Garcia Bartels N, Lünnemann L, Stroux A, Kottner J, Serrano J, Blume-Peytavi U. Effect of diaper cream and wet wipes on skin barrier properties in infants: a prospective randomized controlled trial. Pediatr Dermatol. 2014;31(6):683-91.

27. Brouillette F, Weber ML. Massive aspiration of talcum powder by an infant. Can Med Assoc J. 1978;119(4):354-5.

28. Matina F, Collura M, Maggio MC, Vitulo P, Lo Piparo C, Corsello G. Inhaled surfactant in the treatment of accidental talc powder inhalation: a new case report. Ital J Pediatr. 2011;37:47.

\section{Publisher's Note}

Springer Nature remains neutral with regard to jurisdictional claims in published maps and institutional affiliations.

Ready to submit your research? Choose BMC and benefit from:

- fast, convenient online submission

- thorough peer review by experienced researchers in your field

- rapid publication on acceptance

- support for research data, including large and complex data types

- gold Open Access which fosters wider collaboration and increased citations

- maximum visibility for your research: over $100 \mathrm{M}$ website views per year

At $\mathrm{BMC}$, research is always in progress.

Learn more biomedcentral.com/submissions 